\title{
COMPUTING FENCHEL-NIELSEN COORDINATES IN TEICHMÜLLER SHAPE SPACE
}

\author{
MIAO JIN*, WEI ZENG ${ }^{\dagger}$, NING DING* ${ }^{*}$ XIANFENG GU ${ }^{\dagger}$, AND SHING-TUNG YAU ${ }^{\ddagger}$
}

\begin{abstract}
Teichmüller shape space is a finite dimensional Riemannian manifold, where each point represents a class of surfaces, which are conformally equivalent, and a path represents a deformation process from one shape to the other. Two surfaces in the real world correspond to the same point in the Teichmüller space, only if they can be conformally mapped to each other. Teichmüller shape space can be used for surface classification purpose in shape modeling.

This work focuses on the computation of the coordinates of high genus surfaces in the Teichmüller space. The coordinates are called as Fenchel-Nielsen coordinates. The main idea is to deform the surface conformally using surface Ricci flow, such that the Gaussian curvature is -1 everywhere. The surface is decomposed to several pairs of hyperbolic pants. Each pair of pants is a genus zero surface with three boundaries, equipped with hyperbolic metric. Furthermore, all the boundaries are geodesics. Each pair of hyperbolic pants can be uniquely described by the lengths of its boundaries. The way of gluing different pairs of pants can be represented by the twisting angles between two adjacent pairs of pants which share a common boundary.

The algorithms are based on Teichmüller space theory in conformal geometry, and they utilize the discrete surface Ricci flow. Most computations are carried out using hyperbolic geometry. The method is automatic, rigorous and efficient. The Teichmüller shape space coordinates can be used for surface classification and indexing. Experimental results on surfaces acquired from real world showed the practical value of the method for geometric database indexing, shape comparison and classification.
\end{abstract}

Keywords: conformal geometry, Teichmüller space, shape space, shape analysis, shape classification.

1. Introduction. Surfaces in real life have multiple geometric structures, such as topology, conformal structure, Riemannian metric etc. They can be classified according to different geometric structures. This work focuses on the classification which is based on conformal geometric structures.

A conformal mapping between two surfaces preserves angles. Two surfaces are conformally equivalent, if there exists a conformal mapping between them. All conformal equivalent classes for fixed topology form a finite dimensional Riemannian manifold, the so-called Teichmüller space. In this shape space, each point represents a class of surfaces, and a curve is a deformation process from one shape to the other. Teichmüller theory plays an important role in Riemann surface theory, differential geometry and theoretic physics. With the advancement of computational conformal

*the Center for Advanced Computer Studies, University of Louisiana at Lafayette, Lafayette, LA 70504 USA. E-mail: \{mjin, nding\}@cacs.louisiana.edu

$\dagger$ Department of Computer Science, Stony Brook University, Stony Brook, NY 11794, USA. E-mail: zzengwei,gu\}@cs.sunysb.edu

${ }_{\ddagger}^{\ddagger}$ Mathematics Department, Harvard University, Cambridge, MA 02138, USA. E-mail: yau@math.harvard.edu 
geometry, the coordinates of shapes in Teichmüller space can be computed efficiently today, which is the major focus of the current work.

The main idea for Teichmüller space coordinates is as follows. First, each closed surface of genus $g>1$ in $\mathbb{R}^{3}$ has an induced Euclidean metric. By using curvature flow method, one can conformally deform the metric to a canonical Riemannian metric with constant -1 Gaussian curvature, which is called hyperbolic metric. Then under the hyperbolic metric, one can decompose the surface to $2 g-2$ pairs of pants, ( a pair of pants are a genus zero surface with three boundaries), by cutting the surface along $3 g-3$ geodesic loops. Two adjacent pairs of pants are glued together along a cutting geodesic loop with an angle, called twisting angle. The lengths of the cutting loops and the twisting angles give the coordinates of the surface in the Teichmüller space, which are the so-called Fenchel-Nielsen coordinates.

The Fenchel-Nielsen coordinates uniquely determine the conformal structure of the surface. They can be treated as the fingerprint of the surfaces and can be applied for shape comparison and classification. Two surfaces with the same Fenchel-Nielsen coordinates can be further compared by their Riemannian metrics and the embedding in $\mathbb{R}^{3}$.

Contributions. The major contributions of the current work are:

1. A framework of using Teichmüller shape space for surface classification and comparison.

2. A set of rigorous and practical algorithms for computing Fenchel-Nielsen coordinates for high genus surfaces, including hyperbolic pants decomposition, computing the closed geodesic, shortest paths on hyperbolic pants etc.

The computational algorithms are based on hyperbolic surface Ricci flow. Most computations are carried out using hyperbolic geometric methods. We tested our algorithms using surfaces from real life. Potential applications are illustrated as well.

The paper is organized in the following way: Section 2 will briefly introduce the previous works in the literature; Section 3 will introduce the major theoretic concepts from differential geometry, Riemann surface theory; Details of algorithms are explained in Section 4; experimental results are reported in Section 5; and the work is concluded in Section 6.

2. Previous Works. Our work proposes to compute Teichmüller space coordinates as shape descriptors based on surface hyperbolic uniformization metric, which classifies surfaces according to their conformal structures. Surfaces which have the same descriptors share the same conformal structure, invariant to conformal deformations.

The research literature on shape descriptors is vast. A thorough review of shape descriptors is beyond the scope of current work. We will focus here only on recent shape descriptors which are most relevant to our work using conformal geometry, and 
methods for designing metrics by prescribed curvatures.

2.1. Shape Descriptors. For the application of 3D shape classification and matching, shape descriptors are to extract meaningful and simplified representations from the 3D model based on the geometric and topological characteristics of the object. As the name suggests, shape descriptors should be descriptive enough to be able to distinguish similar and dissimilar shapes. The interested reader is referred to [1], [2] and [3] for comprehensive surveys of different shape descriptors and evaluations of their performance.

Shape descriptors can be classified by the corresponding transformation groups, to which they are invariant. The following transformation groups are considered: rigid motion, isometric transformation and conformal deformation. The former groups are the subgroups of the latter ones. In the discussion, we focus on shape descriptors based on conformal geometry. There are many other shape descriptors invariant to the above transformation groups based on other methods. We only brief some of them.

2.1.1. Shape Descriptors Invariant to Conformal Deformations. Conformal structure is invariant to conformal deformations, which include isometric deformations and rigid motions. To the best of our knowledge, the first work proposed to use conformal structure for shape classification is [4], where the conformal structure is represented as period matrices. Later, geodesic spectrum of surfaces under their uniformization metrics are applied as the conformal structure descriptors in [5], which can be computed symbolically. A general framework for 3D surface matching is proposed in [6] and [7]. By conformally parameterizing the 3D surfaces to canonical 2D domains, the matching problem is greatly simplified. If the surfaces are conformally equivalent, then 2D mapping is an identity with appropriate boundary conditions. Recently, Luo coordinates [8], which define surface conformal structure in Teichmüller space using the lengths of a special group of geodesics on surfaces, are used for shape descriptors in [9].

Previous methods using geodesic lengths as coordinates have more than $6 g-6$ numbers, which is the dimension of Teichmüller space. Therefore there are redundancy. For Fenchel-Nielsen coordinates, each coordinate component is independent of others, so the representation is more compact. The correlations among the components of Luo's coordinates are complicated and unclear, while the F-N coordinates have strong intuition behind. Basically, each pair of hyperbolic pants are determined by their boundary lengths; the twisting angle determine the gluing pattern of pants. So it is trivial to construct a Riemann surface purely from its F-N coordinates. Furthermore, Our method based on consistent hyperbolic pants decomposition has less ambiguity when used for surfaces comparison purpose. 
2.1.2. Shape Descriptors Invariant to Isometric Transformations. Pose changes are a quasi-isometric transformation of the 3D mesh, in the sense that edge lengths do not change much as a result of the transformation. Pose-invariant Shape Descriptors are invariant under non-rigid isometric transformations, and tolerant quasi-isometric transformations. Pose-invariant shape descriptors based on conformal geometry is introduced in [10], where the histogram of the conformal factor computed from surface uniformization metric is applied as shape descriptor. This descriptor is intrinsic and pose-invariant.

Laplace-Beltrami operator is determined by the Riemannian metric. Therefore, most descriptors related to discrete laplace-Beltrami operators are also invariant to isometric deformations, and tolerant quasi-isometric deformations. For examples, Reuter et al. in [11] use the eigenvalues of Laplace-Beltrami operator; Rustamov in [12] uses the eigenvectors; Xiang et al. in [13] use the histogram of the solution to the volumetric Poisson equation which involves the Laplace-Beltrami operator.

2.1.3. Shape Descriptors Invariant to Rigid Motions. Shape descriptors which are invariant to rigid motions and based on conformal geometry are used in [14] and [15] for medical application purpose, where both conformal factor and mean curvature are considered. Conformal factor itself fully determines the Riemannian metric of surfaces. After adding mean curvature, they two can determine the embedding of surfaces unique up to rigid motions with appropriate boundary conditions.

2.1.4. Other Shape Descriptors. There are many other shape descriptors invariant to isometric deformations based on Riemannian geometry. For example, those methods in $[16,17,18]$ compute from surface geodesic distances. The method in [19] computes the diameter of the 3D shape at each point, and the average geodesic distance from each point to all other points. The histograms of the two functions are applied as the shape descriptors.

Many global or local features based, or graph based shape descriptors are invariant to rigid motions, while extra algorithms for feature and graph matching are necessary. We refer readers to [3] for more details.

3. Theoretic Background. This section briefly introduces the background knowledge of conformal geometry, which is necessary for the discussion in the work. The basic concepts of algebraic topology and hyperbolic geometry are briefly introduced in the Appendix also. For more details, we refer readers to the classical textbooks [20], [21] and [22].

3.1. Conformal Structure. Let $S$ be a topological surface, we consider all the possible Riemannian metrics on $S, G=\{\mathbf{g}\}$. Two metrics $\mathbf{g}_{1}, \mathbf{g}_{2}$ are said to be conformally equivalent, $\mathbf{g}_{1} \sim \mathbf{g}_{2}$, if there exists a function $u: S \rightarrow \mathbb{R}$, such that $\mathbf{g}_{1}=e^{2 u} \mathbf{g}_{2}$. 


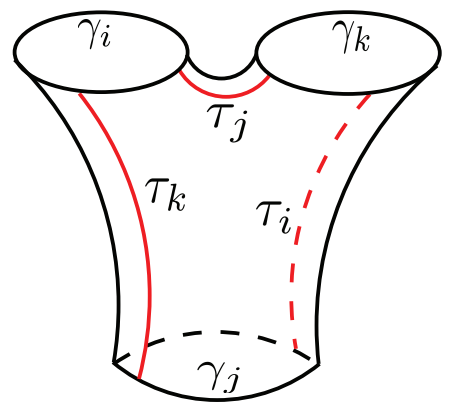

(a) A pair of hyperbolic pants

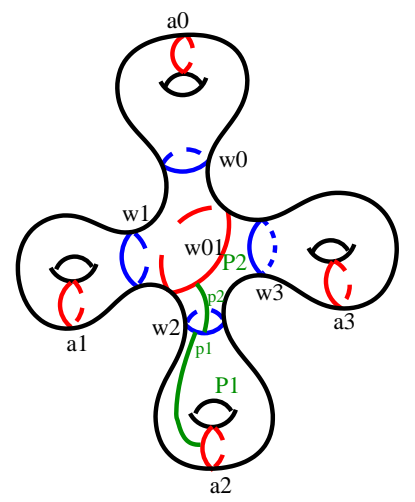

(b) Hyperbolic pants decomposition

FIG. 1. (a) A pair of hyperbolic pants with three geodesic boundaries. (b) A genus g surface with hyperbolic metric is decomposed to $2 g-2$ pairs of hyperbolic pants by $3 g-3$ geodesic cutting loops. The twisting angles and lengths of cutting loops give the Fenchel-Nielsen coordinates in the shape space. Here we visualize the twisting angle on $w_{2}$, which equals to the ratio between the hyperbolic distance of $\left|p_{1}, P_{2}\right|$ and the geodesic length of $w_{2}$.

Intuitively, the angle values measured by conformally equivalent metrics are the same. Hence, conformal means angle preserving. Then each conformal equivalent class of the Riemannian metrics in $G / \sim$ is a conformal structure.

A mapping between two Riemann surfaces $f: S_{1} \rightarrow S_{2}$ is conformal, if it preserves angles. Conformal mappings preserve conformal structures. Namely, if there exists a conformal mapping between $S_{1}$ and $S_{2}$, the $S_{1}$ and $S_{2}$ have the same conformal structure.

3.2. Uniformization Theorem. In each conformal equivalent class of Riemannian metrics, there exists a special metric, that induces constant Gaussian curvature. This is the most fundamental fact for surfaces.

Theorem 3.1 (Uniformization). Let $S$ be a surface with a Riemannian metric $\mathbf{g}$, there exists a Riemannian metric $\tilde{\mathbf{g}}$, such that $\tilde{\mathbf{g}}$ is conformal to $\mathbf{g}$ and induces constant Gaussian curvature, which is one of $\{+1,0,-1\}$.

For surfaces $S$ with negative Euler number, there exists a unique hyperbolic metric conformal to the original metric. The universal covering space of $S$ with the hyperbolic metric can be isometrically embedded in the hyperbolic space $\mathbb{H}^{2}$. All the deck transformations are Möbius transformation. The deck transformation group is called the Fuchsian group of $S$. According to Gauss-Bonnet theorem, each homotopy class has a unique closed geodesic on a surface with a hyperbolic metric.

3.3. Teichmüller Space and Fenchel-Nielsen Coordinates. Let $S$ be a closed topological surface of genus $g>1$. All the conformal structures on $S$ form a $6 g-6$ dimensional manifold, called as Teichmüller space, denoted as $T_{g}$. Because 
each conformal structure has a unique hyperbolic metric, it is enough to consider only surfaces with hyperbolic metrics for computing the Teichüller space.

Assume $S$ is with a hyperbolic metric, then its coordinates in $T_{g}$ can be constructed in the following way.

Definition 3.2 (Pants). A pair of topological pants is a genus zero surface with three boundaries.

Given a genus $g$ surface, it can be decomposed to $2 g-2$ pairs of pants. Figure 1 illustrates one example. Assume all the cutting loops are geodesics $\left\{\gamma_{1}, \gamma_{2}, \cdots, \gamma_{3 g-3}\right\}$, then each pair of pants is pair of hyperbolic pants.

Definition 3.3 (Hyperbolic Pants). A pair of pants is called a pair of hyperbolic pants, if it is with a hyperbolic metric, and all boundaries are geodesics.

For each pair of hyperbolic pants $P$ with three boundaries $\gamma_{i}, \gamma_{j}, \gamma_{k}$, there are three shortest paths connecting each pair of boundaries, e.g. $\tau_{i}$ connects $\gamma_{j}, \gamma_{k}$, and intersects $\gamma_{j}$ and $\gamma_{k}$ with right corner angles.

Suppose two pairs of hyperbolic pants $P_{1}$ and $P_{2}$ are glued together along $\gamma$. The shortest path $\tau_{1}$ on $P_{1}$ intersects $\gamma$ at $p_{1}$, and the shortest path $\tau_{2}$ on $P_{2}$ intersects $\gamma$ at $p_{2}$, then the twisting angle on $\gamma$ is given by

$$
\theta=2 \pi \frac{d\left(p_{1}, p_{2}\right)}{|\gamma|}
$$

where $d\left(p_{1}, p_{2}\right)$ is the geodesic distance between $p_{1}$ and $p_{2},|\gamma|$ is the length of $\gamma$.

Definition 3.4 (Fenchel-Nielsen Coordinates). Suppose $S$ is a genus $g>1$ closed surface with a hyperbolic metric. $S$ is decomposed to pairs of pants $\left\{P_{1}, P_{2}, \cdots\right.$, $\left.P_{2 g-2}\right\}$ by closed geodesics $\left\{\gamma_{1}, \gamma_{2}, \cdots, \gamma_{3 g-3}\right\}$. Then Fenchel-Nielsen coordinates of $S$ in the Teichmüller space $T_{g}$ are given by

$$
\left\{\left(l_{1}, \theta_{1}\right),\left(l_{2}, \theta_{2}\right), \cdots,\left(l_{3 g-3}, \theta_{3 g-3}\right)\right\},
$$

where $\left(l_{k}, \theta_{k}\right)$ are the length and twisting angle of $\gamma_{k}$.

3.4. Surface Ricci Curvature Flow. Let $S$ be a surface embedded in $\mathbb{R}^{3}$. $S$ has a Riemannian metric induced from the Euclidean metric of $\mathbb{R}^{3}$, denoted by g. Suppose $u: S \rightarrow \mathbb{R}$ is a scalar function defined on $S$, then $\overline{\mathbf{g}}=e^{2 u} \mathbf{g}$ is also a conformal metric. The Gaussian curvatures will also be changed accordingly. The Gaussian curvature will become

$$
\bar{K}=e^{-2 u}\left(-\Delta_{\mathbf{g}} u+K\right),
$$

where $\Delta_{\mathrm{g}}$ is the Laplacian-Beltrami operator under the original metric $\mathrm{g}$. The above equation is called the Yamabe equation. Yamabe equation can be solved using Ricci flow method. The Ricci flow deforms the metric $\mathbf{g}(t)$ according to the Gaussian curvature $K(t)$ (induced by itself), where $t$ is the time parameter

$$
\frac{d g_{i j}(t)}{d t}=2(\bar{K}-K(t)) g_{i j}(t)
$$




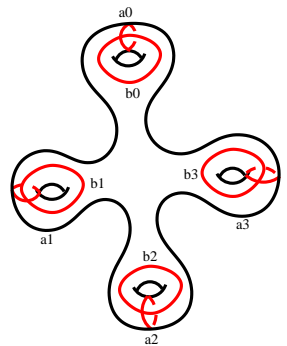

(a)

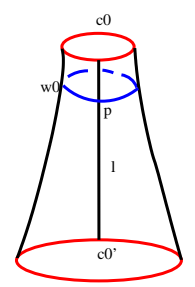

(d)

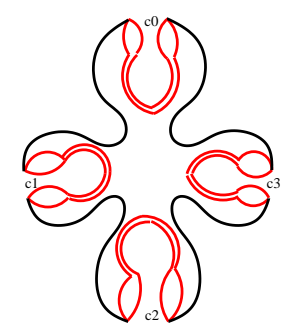

(b)

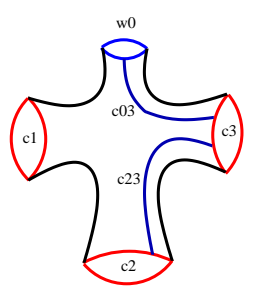

(e)

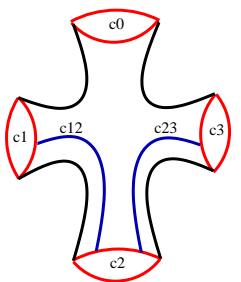

(c)

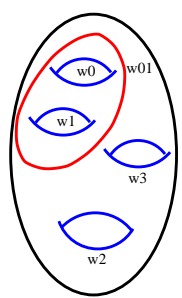

(f)

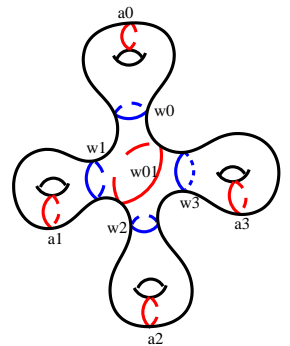

(g)

FIG. 2. Topological pants decomposition for surface with g handles: (a) Compute surface handle loops and tunnel loops; (b) Slice surface open along tunnel and handle loops; (c) Connect all other boundaries except $c_{0}$ to form a big boundary and get a topological cylinder; (d) Find a locally shortest loop $w_{0}$ along the path $l$ connecting boundaries $c_{0}$ and $c_{0}^{\prime}$, which is the waist of the handle; (e) Repeat the process to find waists for each handle; ( $f$ ) Cutting handles out along each waist, we get a topological sphere with g holes. Repeat this process as long as the total number of boundaries is less than 4: a locally shortest loop $w_{i j}$ which is homotopic to $w_{i} \circ w_{j}$ is computed, and surface patch bounded by $w_{i}, w_{j}$, and $w_{i j}$ is cut out. ( $g$ ) The set of cutting loops are tunnel loops computed in (a), waists computed in (e), and loops computed in ( $f)$. They decompose the surface to topological pants.

Ricci flow method can be applied to compute surface hyperbolic metric.

4. Algorithms. The key of our algorithm is to compute hyperbolic pants decomposition for a given closed high genus surface based on surface hyperbolic metric. The geodesic lengths of cutting loops which segment the surface into pairs of hyperbolic pants and the angles of gluing pair of pants together are the Fenchel-Nielsen coordinates of the surface. The pipelines of our algorithms can be listed as:

1. Compute topological pants decomposition (section 4.1);

2. Compute the hyperbolic metric using Ricci flow (section 4.2);

3. Compute hyperbolic pants decomposition (section 4.3);

4. Compute the Fenchel-Nielsen coordinates (section 4.4).

4.1. Compute Topological Pants Decomposition. To get hyperbolic pants decomposition, we need to get topological pants decomposition first. Surface topological pants decomposition has been widely studied [23] and done with the optimal 
segmentation of a given surface into pants [24]. Since the major application of computing Fenchel-Nielsen coordinates in out paper is for surface index and classification purpose, we adopt the methods in [25] and [26] to consistently decompose surfaces with same topology to a set of corresponding pants, which will induce consistent hyperbolic pants decomposition and Fenchel-Nielsen coordinates. For a closed $g>1$ surface, the set of cutting loops which decompose surface to topological pants is $3 g-3$.

The algorithm to consistently decompose surfaces to topological pants can be illustrated in Figure 2.

1. Compute handle and tunnel loops for a given surface (Figure 2(a)): A closed embedded surface $\mathrm{M}$ with genus $g$ in $R^{3}$ separates $R^{3}$ into a bounded space I and an unbounded space O. A loop $b_{i}$ is a handle if it spans a disk in the bounded space I; if one cuts $\mathrm{M}$ along $b_{i}$ and fills the boundary with that disk, one eliminates a handle. A loop $a_{i}$ is a tunnel if it spans a disk in the unbounded space $\mathrm{O}$, and its removal eliminates a tunnel. Tunnel loops and handle loops can be effectively computed using the technique (and the software) presented in [25]. Since handles of given surfaces have been indexed, we will get a set of labeled handle and tunnel loops.

2. each surface handle $h_{i}$ is sliced open along its handle loop $b_{i}$ and tunnel loop $a_{i}$, with the boundary curve $c_{i}=a_{i} \dot{b}_{i} \dot{a}_{i}^{-1} \dot{b}_{i}^{-1}$ (Figure 2(b)).

3 . To compute the waist $w_{i}$, the shortest loop which is homotopic to $c_{i}$, we connect all other $c_{j}$ s to form a large boundary loop $c_{i}$, then we get a topological cylinder (Figure 2(c)). A shortest path $l$ which connects the two boundaries $c_{i}$ and $\dot{c}_{i}$ is computed, then the waist $w_{i}$ is the shortest loop along $l$ (Figure $2(\mathrm{~d})$ ). Then the handle bounded by $c_{i}$ and $w_{i}$ can be cut off, and $w_{i}$ is replaced with $c_{i}$ (Figure 2(e)). We repeat this process until we finish the computation of waists for all handles. Now the surface M is a topological sphere with $\mathrm{g}$ holes( $\mathrm{g}$ is the handle number of the surface).

4. If $g>3$, then for each pair of $w_{i}$ and $w_{j}$ (from the increasing number of indexes), we compute the shortest loop $w_{i j}$ which bounds $w_{i}$ and $w_{j}$. After removing the pant with boundaries $w_{i j}^{\prime}, w_{i}$ and $w_{j}$, we repeat this step until the number of boundaries is less or equal to 3 (Figure 2(f)).

All the tunnel loops computed in the first step, waists computed in the third step, and loops computed in the forth step form the set of cutting loops which segment the given surface to topological pants (Figure $2(\mathrm{~g})$ ). Since we have indexed surfaces handles, the ordered set of topological pants is consistent with surfaces of same topology.

4.2. Compute the Hyperbolic Metric. For a negative Euler number surface, there exists a unique hyperbolic metric conformal to its original metric. The computation of the hyperbolic metric on a triangular mesh is based on the discrete hyperbolic 
Ricci flow algorithm. We brief the outline of the algorithm, details can be found in [27] and [28].

Let $M$ be a simplicial complex (triangular mesh) with vertex set $V$, edge set $E$ and face set $F$. The outline of the algorithm can be listed as the following steps.

1. For each vertex $v_{i}$, assign a circle with the initial radius $r_{i}$ which approximates the original Euclidean metric of the edges associated with the vertex; For each edge $e_{i j}$, its weight $\phi_{i j}$ is the intersection angle of the two circles associated with the ending vertices of the edge, $v_{i}$ and $v_{j}$.

2. The edge length $l_{i j}$ of $e_{i j}$ is updated by current vertex radius and edge weight using the hyperbolic cosine law,

$$
\cosh l_{i j}=\cosh r_{i} \cosh r_{j}+\sinh r_{i} \sinh r_{j} \cos \phi_{i j} .
$$

3. Update the angle $\theta_{i}^{j k}$, related to each corner ${ }_{i} L_{j}^{k}$, using current edge lengths with the inverse hyperbolic cosine law:

$$
\theta_{i}^{j k}=\cos ^{-1} \frac{\cosh \left(l_{i j}\right) \cosh \left(l_{k i}\right)-\cosh \left(l_{j k}\right)}{\sinh \left(l_{i j}\right) \sinh \left(l_{k i}\right)},
$$

4. Compute the discrete Gaussian curvature $K_{i}$ of each vertex $v_{i}$ :

$$
K_{i}=\left\{\begin{array}{cl}
2 \pi-\sum_{f_{i j k} \in F} \theta_{i}^{j k}, & \text { interior vertex } \\
\pi-\sum_{f_{i j k} \in F} \theta_{i}^{j k}, & \text { boundary vertex }
\end{array}\right.
$$

where $\theta_{i}^{j k}$ represents the corner angle attached to vertex $v_{i}$ in the face $f_{i j k}$.

5. Update the radius $r_{i}$ of each vertex $v_{i}$ :

$$
r_{i}=r_{i}+\epsilon\left(\bar{K}_{i}-K_{i}\right) \sinh r_{i}
$$

where $\bar{K}_{i}$ is the target vertex curvature.

6. Continue the procedure from $B$ to $E$, until $\left\|\bar{K}_{i}-K_{i}\right\|$ of all vertices satisfy the user-specified error tolerance.

4.3. Compute Hyperbolic Pants Decomposition. The key to decompose surfaces to hyperbolic pants is to compute geodesics homotopic to the set of cutting loops which decompose the given surface to topological pants (see Section 4.1) under hyperbolic metric (see Section 4.2). Our main idea is to embed the universal cover of the given surface to hyperbolic space, then the set of cutting loops will be mapped to a set of paths. For each path, its two ending points will be projected to the same point on the surface, while in the universal cover, the two ending points for each path induce a Möbius transformation. The axis of each Möbius transformation, when projected from universal cover to the surface, is a geodesic loop homotopic to the original cutting loop. The details of our algorithms are introduced in the following pipelines. 


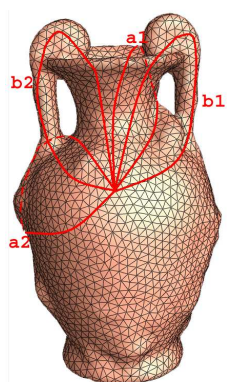

(a)

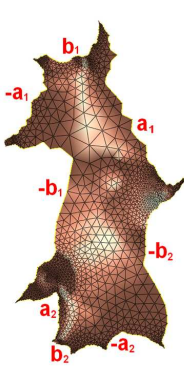

(b)

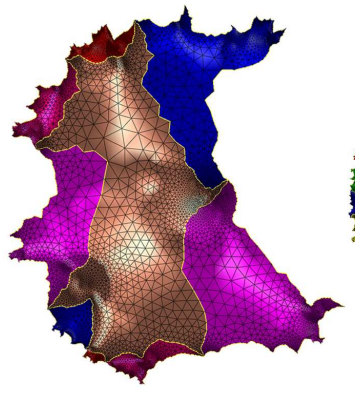

(c)

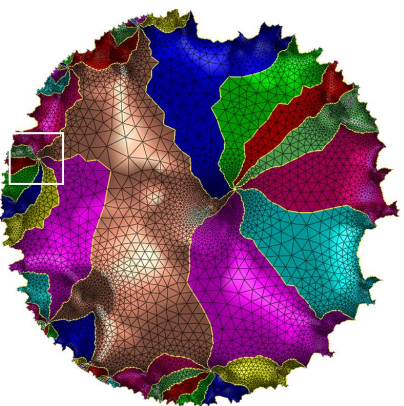

(d)

FIG. 3. Embed the Universal Cover Isometrically onto $\mathbb{H}^{2}$ : (a) A set of canonical homology basis is marked on surface with red; (b) surface is sliced open along homology basis to form a unit disk, the fundamental domain; (c) one layer copies of the fundamental domain are transformed with Möbius transformation and glued with the original one; (d) a portion of the universal cover embedded in Poincaré disk.

4.3.1. Embed the Universal Cover Isometrically onto $\mathbb{H}^{2}$. The major steps to embed the universal cover of a given surface with hyperbolic metric onto $\mathbb{H}^{2}$ are similar with algorithms in [28]. While in this paper, we only need to construct a portion of the universal cover which are needed in the next step, instead of computing all the Fuchsian group transformations.

1. Slice $M$ open along a set of canonical homology basis $a_{1}, b_{1}, a_{1}^{-1}, b_{1}^{-1} \cdots a_{g}, b_{g}$, $a_{g}^{-1}, b_{g}^{-1}$ to form a topological disk, the fundamental domain $\bar{M}$ (Figure $3(\mathrm{a})(\mathrm{b}))$ (see Appendix for definition of canonical homology basis).

2. Embed the seed triangle $f_{012}$ (random chosen from $\bar{M}$ ) into Poincaré disk with positions of the three vertices:

$$
\tau\left(v_{0}\right)=(0,0), \tau\left(v_{1}\right)=\tanh \frac{l_{01}}{2}, \tau\left(v_{2}\right)=\tanh \frac{l_{02}}{2} e^{i \theta_{0}^{12}} .
$$

3. Put all the neighboring faces of the seed face to a queue.

4. Suppose that $f_{i j k}$ is a non-embedded face which is popped out from the queue. If $v_{i}$ and $v_{j}$ have been embedded, $\tau\left(v_{k}\right)$ can be computed as the intersection point between two hyperbolic circles under proper orientation, with centers $\left(\tau\left(v_{i}\right)\right.$ and $\left(\tau\left(v_{j}\right)\right.$, the positions of the two vertices, radii $\left.l_{k i}\right)$ and $\left.l_{k j}\right)$, the edge lengths of $e_{k i}$ and $e_{k j}$ in Poincaré disk. Then we put all the neighboring faces of $f_{i j k}$ into queue.

5. Repeat step D until the queue is empty. Then we get the embedding of the fundamental domain of $M$ onto $\mathbb{H}^{2}$, with boundary segments (Figure 3(b))

$$
\partial \bar{M}=a_{1} b_{1} a_{1}^{-1} b_{1}^{-1} \cdots a_{g} b_{g} a_{g}^{-1} b_{g}^{-1} .
$$

6. To construct the universal cover of $M$, the embedding of infinite copies of $\bar{M}$ in Poincaré disk, we need to compute a set of Möbius transformations, the 


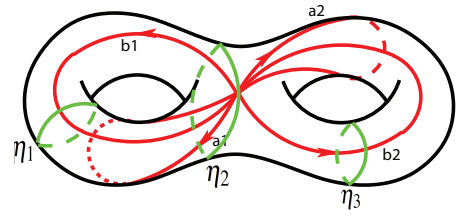

(a)

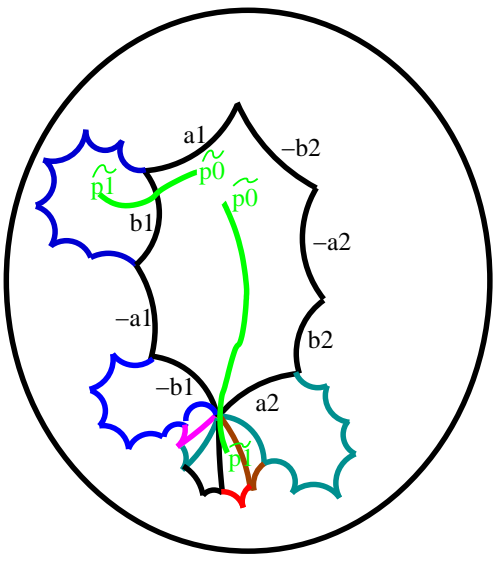

(b)

FIG. 4. Cutting loops which decompose surface to consistent topological pants are lifted to universal cover. (a) We show a set of cutting loops marked with green, and a set of canonical homology basis marked with red on surface; (b) Surface is cut open along homology basis to form a fundamental domain and embedded onto the Poincaré disk. To lift $\eta_{1}$ and $\eta_{2}$ to Poincaré disk, we start from the base point, $p_{0}$ for each loop, and extend vertex by vertex. We have to glue other copies of fundamental domains when we go to the boundary of the center fundamental domain. The worst time complexity case is when we hit a corner point of the fundamental domain, like to lift $\eta_{2}$ to $\tilde{\eta_{2}}$, we have to glue instead of one domain but $4 g-1$ domains.

so called deck transformations, which transform one copy of $\bar{M}$ in Poincaré disk and match with the original copy along the mate boundaries, $a_{i}$ and $a_{i}^{-1}$, or $b_{i}$ and $b_{i}^{-1}$. Suppose we want to transform a copy of $\bar{M}$ by a Möbius transformation $\phi$ along $a_{1}$ of $\bar{M}$. Let the ending points of $a_{1}$ are $p_{1}, q_{1}$ on $\bar{S}$, the ending points of $a_{1}^{-1}$ are $p_{2}, q_{2}$. To find a Möbius transformation $\phi$ which maps $p_{1}, q_{1}$ to $p_{2}, q_{2}$, we first construct a unique Möbius transformation $\phi_{1}$ :

$$
\psi_{0}(\mathbf{z})=e^{-i \theta_{0}} \frac{\mathbf{z}-\mathbf{p}_{0}}{1-\overline{\mathbf{p}}_{0} \mathbf{z}}, \text { where } \theta_{0}=\arg \frac{\mathbf{q}_{0}-\mathbf{p}_{0}}{1-\overline{\mathbf{p}}_{0} \mathbf{q}_{0}},
$$

such that $p_{1}$ is mapped to the origin, and $q_{1}$ to a positive real number. Similarly, we construct another unique Möbius transformation $\phi_{2}$, which maps $p_{2}$ to the origin and $q_{2}$ to a positive real number. Then $\phi=\phi_{2}^{-1} \circ \phi_{1}$.

7. We can repeat this process and glue copies of the fundamental domain along their mate boundaries. Figure 3(c) and (d) shows the process of gluing copies of the fundamental domain of a genus two surface to form a portion of its universal cover embedded in the Poincaré disk. Different fundamental domains are encoded by different colors.

\subsubsection{Compute Hyperbolic Cutting Loops Based on Universal Cover.}

We have computed a set of cutting loops which decompose the given surface to topological pants in Section 4.1. In this section, we propose the algorithms that compute 


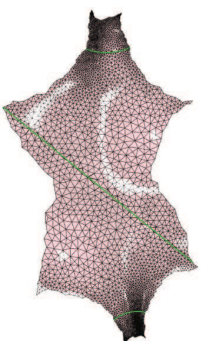

(a)

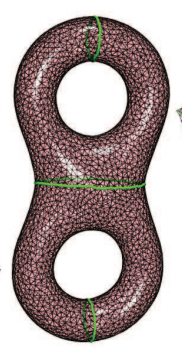

(b)

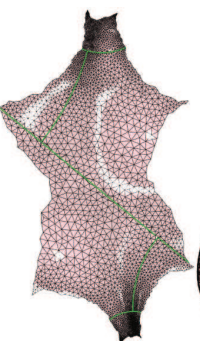

(c)

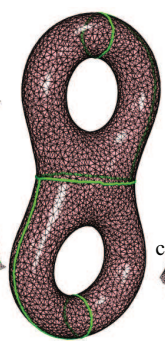

(d)

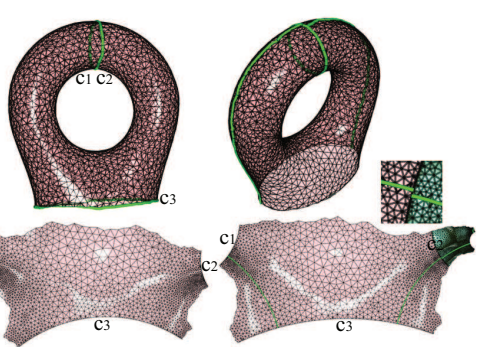

(e)

(f)

FIG. 5. Pipeline of computing Fenchel-Nielsen coordinates: The fundamental domain of the eight surface is embedded in hyperbolic disk as shown in (a). The three geodesics on its domain, when lifted on to the surface as shown in (b), will decompose the eight surface to two hyperbolic pants. One pant is shown in (e), and its boundaries, $c_{1}, c_{2}$, andc3 are geodesics in hyperbolic disk. We compute the geodesics perpendicular to the boundaries of the two pants, and get intersection points. One is shown in (d), and the other is shown in ( $f)$. The twisting angle can be computed from the distance of the two intersection points along the same cut loop. For this eight model, since it is very symmetric, all its twisting angles are close to zero. As visualized in (f), the distance between the two intersection points is very small, almost coincide. Both the three geodesic lengths in (a) and the twisting angles are Fenchel-Nielsen coordinates.
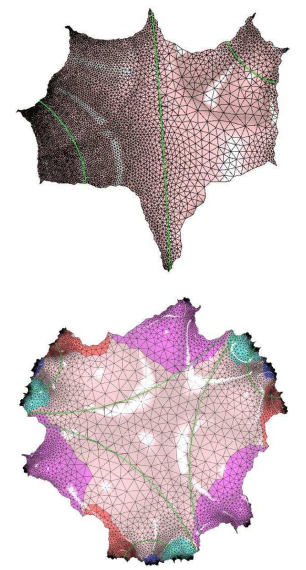

(a)
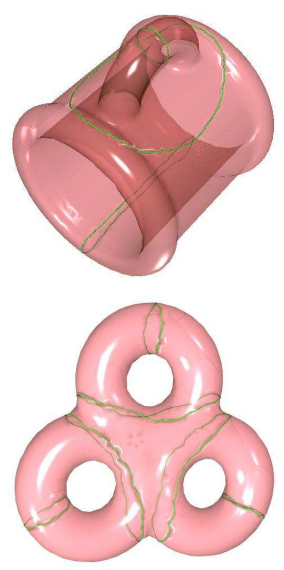

(b)
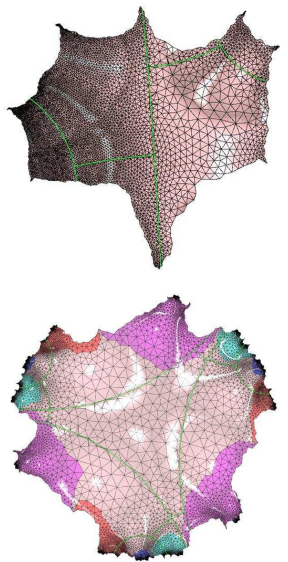

(c)
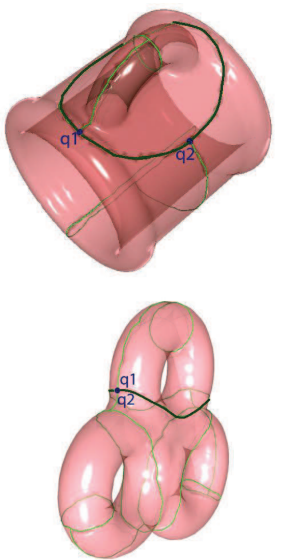

(d)

FIG. 6. Compute the Fenchel-Nielsen Coordinates. (a) Hyperbolic cutting loops computed on universal cover for cup model and 3-hole model; (b) hyperbolic cutting loops computed on universal cover are projected back to original surfaces, and decompose cup model to 2 hyperbolic pants, 3hole model to 4 hyperbolic pants; (c) computing the geodesics perpendicular to the boundaries of these pants on universal cover; (d) those perpendicular geodesics are projected back to the surface to visualize the twisting angles: the ratio between the distance of the two intersection points $\left(q_{1}\right.$ and $q_{2}$ ) along the same cutting loop and the geodesic length of that loop (marked with dark green). Again, the 3-hole surface is very symmetric, so its twisting angle is very small, while for the cup model, its twisting angle is around $\frac{\pi}{2}$. 
a set of geodesics which are homotopic to the set of topological cutting loops and decompose the given surface to hyperbolic pants based on universal cover.

For each cutting loop $\eta$ computed in Section 4.1, we perform a "lifting" process which lifts the loop to the universal cover. In practice, to save space, the lifting is only needed to perform in a finite portion of the universal cover, which contains $\tilde{\eta}$. The portion is constructed during the lifting process "on the fly", which means we glue one more copy of the fundamental domain only if we have to. The steps of the "lifting" can be summarized as:

1. For one cutting loop $\eta$ on surface $M$, we choose one point $p$ ( can be arbitrary point in $\eta$ ) as the base point of the loop.

2. To lift $\eta$ to universal cover, We first lift the base point $p$ to the center fundamental domain $\bar{M}$.

3. Then we lift next vertex connecting $\mathrm{p}$ through edge $e_{p}$ along the loop under $\mathrm{CCW}$ direction. we extend the lifting vertex by vertex. Whenever the lifted loop intersects the boundary segment of the fundamental domain, we compute a Möbius transformation (with the method in Section 4.3.1) to glue a new copy of the fundamental domain along that boundary segment, then we continue the extension of the lifting (see $\tilde{\eta_{1}}$ in Figure 4(b)). If the lifted path goes through a corner point of the fundamental domain, we need to compute $4 g-1$ Möbius transformations and glue $4 g-1$ copies at that corner (see $\tilde{\eta_{2}}$ in Figure 4(b)).

4. When the lifting process comes back to the edge $e_{p}$, we have lifted the cutting loop $\eta$ in $M$ to a path $\tilde{\eta}$ in universal cover, with the base point lifted to the two points $\tilde{p}_{0}$ and $\tilde{p}_{1}$, and edge $e_{p}$ lifted to two edges $\tilde{e_{p_{0}}}$ and $\tilde{e_{p_{1}}}$ of $\tilde{\eta}$.

5. Similarly, We can construct a deck transformation $\tau$, such that $\tau\left(\tilde{e_{p_{0}}}\right)=\tilde{p_{p_{1}}}$.

6. Since $\tau$ is a Möbius transformation, its two fixed points can be computed as

$$
s=\lim _{n \rightarrow \infty} \tau^{n}(z), t=\lim _{n \rightarrow \infty} \tau^{-n}(z),
$$

where $z$ is an arbitrary point in the unit disk.

7. A unique geodesic $\tilde{\gamma}$ in Poincaré disk passing through $s$ and $t$ can be computed, which is the axis of $\tau$.

Then the projection of $\tilde{\gamma}, \gamma=h(\tilde{\gamma})$, from universal cover back to the original surface, is the geodesic homotopic to $\eta$.

4.4. Compute the Fenchel-Nielsen Coordinates. Let the geodesic cutting loops computed from Section 4.3 .2 be $\left\{\gamma_{1}, \gamma_{2}, \cdots, \gamma_{3 g-3}\right\}$, we can decompose surface $M$ to hyperbolic pants. For a pair of hyperbolic pants $S$, the three boundaries $\partial S=$ $\gamma_{i}+\gamma_{j}+\gamma_{k}$ are geodesics in hyperbolic space. Since we have indexed each handle at the step of topological pants decomposition, we will consistently assign a number to each boundary of the pant. 
To compute the Fenchel-Nielsen coordinates, we first compute the length of each geodesic cutting loop. They can be easily computed using hyperbolic geometry. Here are the steps:

1. For each geodesic cutting loop $\gamma_{k}$ on $M$, the same as we lift the topological cutting loop to universal cover in Section 4.3.2, we first choose one base point $p$ on that loop, then lift that base point to universal. We extend the lifting vertex by vertex along this loop until we are back to the base point.

2. Then $\gamma_{k}$ is lifted to universal cover as part of a geodesic hyperbolic line, with $p$ lifted to $\tilde{p}_{0}$ and $\tilde{p}_{1}$. The geodesic hyperbolic line will intersect the unit circle at $q_{0}$ and $q_{1}$, then the length of $\gamma_{k}$ is given by the logarithm of the cross ratio of $\left\{q_{0}, \tilde{p}_{0}, \tilde{p}_{1}, q_{1}\right\}$.

To compute the twisting angle associated with each geodesic cutting loop, the algorithm is:

1. Suppose geodesic cutting loop $\gamma_{k}$ glues the two pairs of pants $P_{1}$ and $P_{2}$ together. The lifting of $\gamma_{k}$ and other boundaries of pants $P_{1}$ and $P_{2}$ (other geodesic cutting loops) are geodesic hyperbolic lines in Poincaré disk.

2. The geodesic $\zeta_{1}$ between $\tilde{\gamma_{k}}$ and $\tilde{\gamma_{1}}$ (let $\tilde{\gamma_{1}}$ be one of the other two lifted boundaries of pant $P_{1}$, with the smallest assigned number) is also a hyperbolic line in Poincaré disk, which is not only perpendicular to $\tilde{\gamma_{k}}$ and $\tilde{\gamma_{1}}$, but also perpendicular to the unit circle. Namely, we compute a circular arc, orthogonal to three circles, the unit circle, $\tilde{\gamma_{k}}$, and $\tilde{\gamma_{1}} \cdot \zeta_{1}$ is unique.

3. The same we compute the geodesic $\Upsilon_{2}$ between $\tilde{\gamma_{k}}$ and $\tilde{\gamma_{2}}$ (the lifted boundary of pant $P_{2}$ ).

4. Suppose $\zeta_{1}$ intersects $\gamma_{k}$ with point $q_{1}$, and $\zeta_{2}$ intersects $\gamma_{k}$ with point $q_{2}$, hyperbolic distance between $q_{1}$ and $q_{2}$ is $\left|q_{1} q_{2}\right|$, then the twisting angle is given by

$$
\theta_{k}=2 \pi \frac{\left|q_{1} q_{2}\right|}{l_{k}}
$$

where $l_{k}$ is the length of $\tilde{\gamma_{k}}$ in Poincaré disk.

Then the Fenchel-Nielsen coordinates are given by

$$
\left\{\left(l_{1}, \theta_{1}\right),\left(l_{2}, \theta_{2}\right), \cdots,\left(l_{3 g-3}, \theta_{3 g-3}\right)\right\} .
$$

Figures 5 and 6 visualizes the computation of Fenchel-Nielsen coordinates and results on models with different topologies.

5. Experimental Results. In our experiments, most of our testing surfaces are closed genus two surfaces. For closed genus two surfaces, the dimension of FenchelNielsen coordinates is six. Half are lengths of geodesics, and half are associated twisting angles. Due to the page limit, Table 1 only lists the coordinates of some genus two surfaces in our experiments. 
Table 2 gives part of our experimental results. For a pair of surfaces, the first number is their geodesic length difference, and the second number is their angle difference. By a quick check of this table, we can easily find the most similar and the most non-similar surfaces with the selected retrieval surface in terms of their conformal structure. For example, Table 3 shows the neighborhoods of the selected surfaces and also surfaces far away from them in the Teichmüller space.

We use L2 norm to measure their angle differences and geodesic length differences to approximate their geodesic distances in Teichmüller space. So we can classify surfaces based on their conformal structure. Figure 7 shows the clustering of those genus two surfaces, with the x-coordinate representing the twisting angle, the $y$ coordinate representing the geodesic length. Based on twisting angles, we can classify them into three big groups. Then we can get more refined groups with marked circles after adding geodesic lengths.

We compare our method with other existing conformal structure based methods. The results are offered in Table 4 with a selected teapot and its distances to the other teapots. The sorting result of our method is same with using Luo coordinates in [9], while there is no redundancy of coordinates in our method, also, our consistent hyperbolic pants decomposition can guarantee easily the consistent comparison of coordinates for surfaces with same topology.

Since the time complexities of algorithms to compute consistent topological pants decomposition and surface hyperbolic metric have been reported in [26] and [28] respectively, we only analyze the time complexities of algorithms to compute hyperbolic pants decomposition and Fenchel-Nielsen coordinates. Although the time complexity to construct universal cover grows exponentially with surface genus number, and the computation of both hyperbolic cutting loops and Fenchel-Nielsen coordinates are based on universal cover, we do not need to compute all the Möbius transformations and glue all the copies of the domain. When we lift the topological cutting loops to universal, we start from one center domain in Poincaré disk, then glue another domain only when the extension process hits the boundary of the center domain. The worst case is that we hit the corner points of the domain, then we have to compute $4 g-1$ Möbius transformations and glue that number of copies of domain to Poincaré disk. Since we have $3 g-3$ cutting loops for a surface with genus $g$, and for each cutting loop $\eta$, suppose $|\eta|$ is its word length in $\pi_{1}(S, p)$ (see Appendix), the time complexity to construct universal cover is $(3 g-3) *(4 g-1) *|\eta|$. For other computations, they are linear to the number of vertices and edges of the surface.

6. Conclusion. This paper introduces the computational algorithms for Fenchel-Nielsen coordinates for closed high genus surfaces in the Teichmüller space. The method is based on Teichmüller space theory, which is automatic, rigorous and efficient. Details of the algorithm has been thoroughly explained. Computational 
TABLE 1

Consistent Fenchel-Nielsen coordinates of genus two surfaces: the length of each geodesic and the twisting angle associated with that geodesic.

\begin{tabular}{|c|c|c|c|c|c|c|}
\hline Distance & \multicolumn{3}{|c|}{ Geodesic Length } & \multicolumn{3}{|c|}{ Twisting Angle } \\
\hline & 1.542 & 4.07 & 1.536 & 0.01 & 0.05 & 0.01 \\
\hline & 1.52 & 4.443 & 1.844 & 0.002 & 1.495 & 0.001 \\
\hline & 7.160 & 4.202 & 0.180 & 0.005 & 1.507 & 0.005 \\
\hline & 0.706 & 3.957 & 0.343 & 0.001 & 3.115 & 0.001 \\
\hline
\end{tabular}

efficiency has also been reported.

Fenchel-Nielsen coordinates can be used to compare and classify surfaces based on their conformal structures, and can also help to understand the structure of surfaces, like their symmetry information. For example, the twisting angle gives a quantitative way to measure how two pants are glued together, like the two pants of the cup model in Figure 6(b) glued with a $\frac{\pi}{2}$ angle twisting. We will explore the direction further.

Although our current algorithms focus on closed high genus surfaces, they can also be applied directly to surfaces with boundaries, as long as the Euler number of the surface is negative. We will include these cases in our future research.

In this paper, we use Euclidean distances between consistent Fenchel-Nielsen coordinates of surfaces to approximate their distance in Teichmüller space. We will explore feasible algorithms to compute the real geodesics in Teichmüller space.

In the future, we plan to further test our algorithm for large scale geometric database indexing and many other real applications in engineering fields.

Appendix. In the appendix, we briefly introduce the concepts from algebraic topology and hyperbolic geometry, which are essential for understanding and implementing the algorithm described in the current work. 
TABLE 2

Difference of Fenchel-Nielsen coordinates on surfaces: the first number is the geodesic length difference; the second number is the twisting angle difference; both of them contribute to the distance between surfaces in Teichmüller Space.

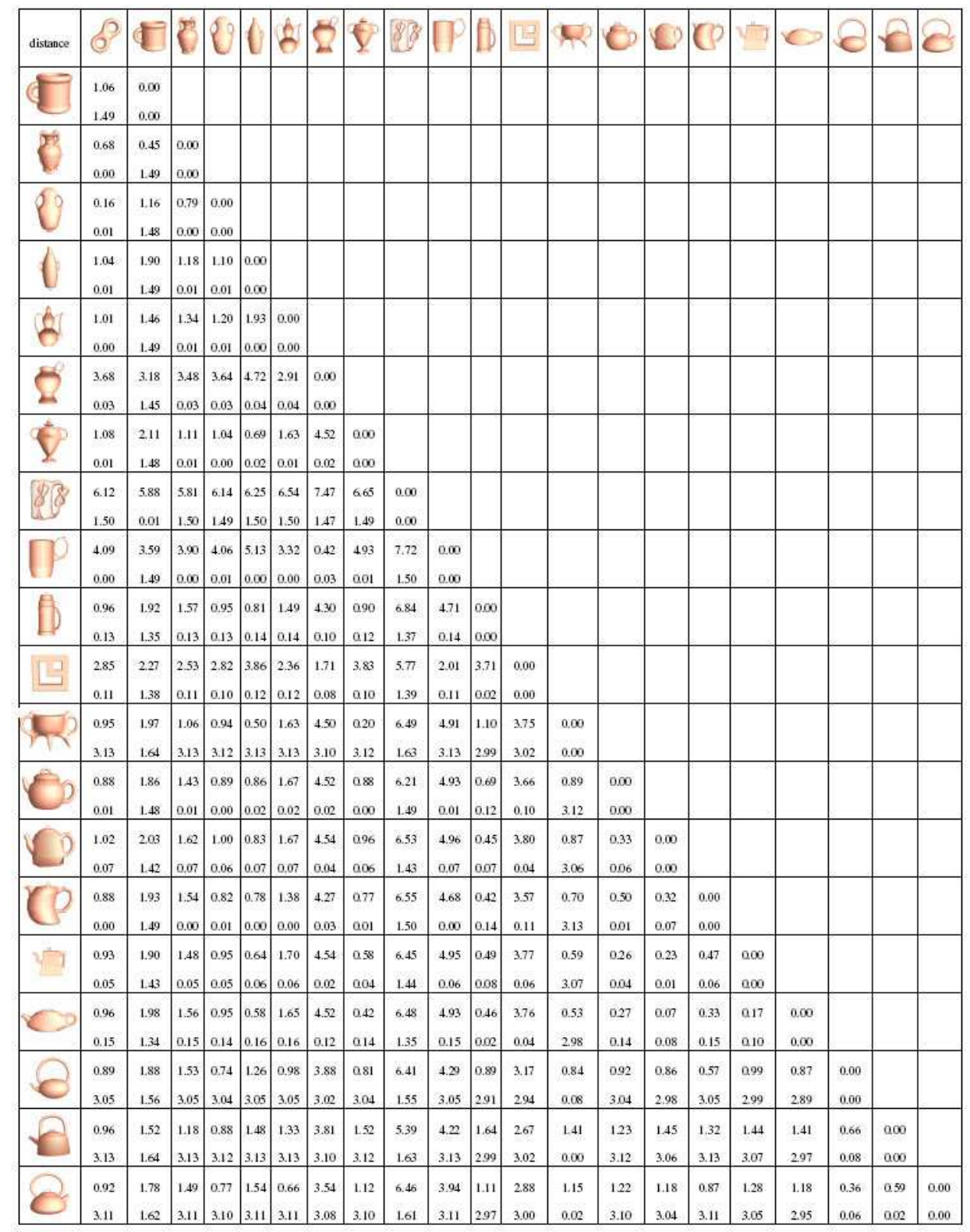

6.1. Fundamental Group and Representation of Homotopy Class. Let $S$ be a topological surface, and let $p$ be a point of $S$. We are interested in the set of continuous functions $f:[0,1] \rightarrow S$ with the property that $f(0)=p=f(1)$. These functions are called loops with base point $p$. Any two such loops, say $f$ and $g$, are 
TABLE 3

The sorted distances based on Fenchel-Nielsen coordinates between selected models and other models in Techmüller space by checking table I. Here we only show models with maximum and minimum distances. Again, the first number indicates the geodesic length difference, and the second number indicates the twisting angle difference.

\begin{tabular}{|c|c|c|c|c|}
\hline Distance & & & & 88 \\
\hline \multirow[b]{3}{*}{ Distance } & 0.32 & 0.33 & 4.68 & 6.55 \\
\hline & 0.07 & 0.15 & 0.00 & 1.50 \\
\hline & & $\square$ & & \\
\hline \multirow{2}{*}{$\theta$} & 0.42 & 1.71 & 4.50 & 7.47 \\
\hline & 0.03 & 0.08 & 3.10 & 1.47 \\
\hline
\end{tabular}

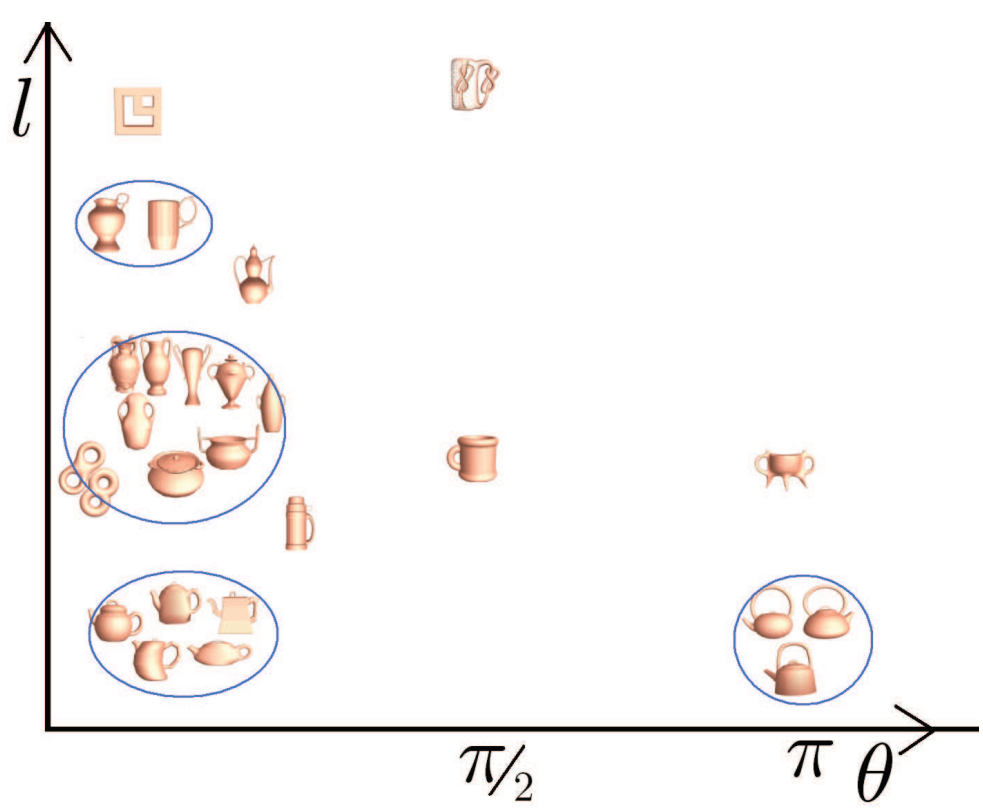

FIG. 7. Clustering of surfaces based on their Fenchel-Nielsen coordinates. The $x$-coordinate indicates the twisting angle, and the $y$-coordinate indicates the geodesic length. Surfaces are clustered based on both their twisting angle and geodesic lengths, with different groups marked with circles.

considered equivalent if there is a continuous function $h:[0,1] \times[0,1] \rightarrow S$ with the property that, for all $0 \leq t \leq 1, h(t, 0)=f(t), h(t, 1)=g(t)$ and $h(0, t)=p=h(1, t)$. Such a $h$ is called a homotopy from $f$ to $g$, and the corresponding equivalence classes 
TABLE 4

Comparison: sorted distances between selected teapot model and other teapot models using different conformal invariant based methods. Since the dimension of both geodesic spectrum and conformal factors on surfaces are infinite, we choose the lengths of the first 14 sorted geodesics as shape descriptors for geodesic spectrum based method, and for conformal factors based method, their histgrams in finite range are used to do comparison.

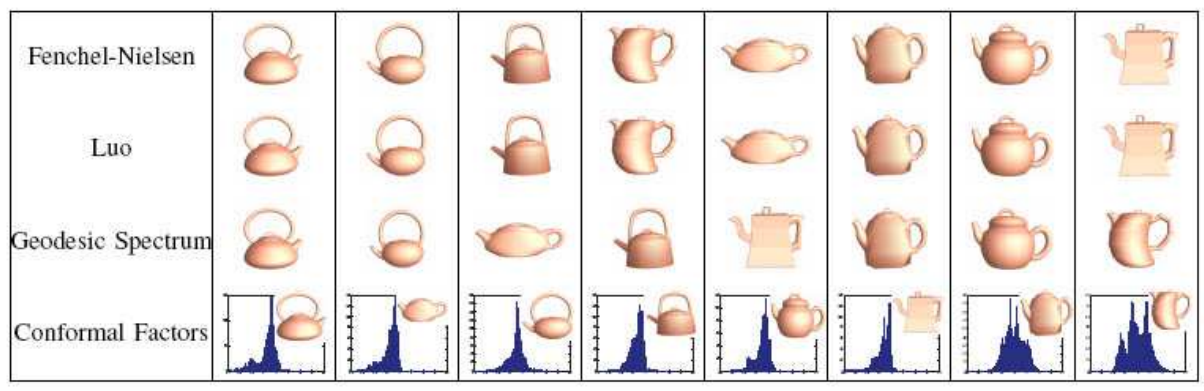

are called homotopy classes.

The product $f \cdot g$ of two loops $f$ and $g$ is defined by setting $(f \cdot g)(t):=f(2 t)$, if $0 \leq t \leq 1 / 2$ and $(f \cdot g)(t):=g(2 t-1)$ if $\frac{1}{2} \leq t \leq 1$. The product of two homotopy classes of loops $[f]$ and $[g]$ is then defined as $[f \cdot g]$, and it can be shown that this product does not depend on the choice of representatives.

With the above product, the set of all homotopy classes of loops with base point $p$ forms the fundamental group of $S$ at the point $p$ and is denoted $\pi_{1}(S, p)$. The identity element is the constant map at the base point, and the inverse of a loop $f$ is the loop $g$ defined by $g(t)=f(1-t)$.

Suppose $S$ is a genus $g$ closed surface. A canonical set of generators of $\pi(S, p)$ consists of $\left\{a_{1}, b_{1}, a_{2}, b_{2}, \cdots, a_{g}, b_{g}\right\}$, such that the pair $a_{i}$ and $b_{i}$ has one intersection point, the pairs $\left\{a_{i}, a_{j}\right\},\left\{b_{i}, b_{j}\right\}$ and $\left\{a_{i}, b_{j}\right\}$, have no intersections, where $i \neq j$. See figure 3(a) for an example of canonical basis on a genus two surface.

6.2. Universal Cover and Uniformization Metric. A covering space of $S$ is a space $\tilde{S}$ together with a continuous surjective map $h: \tilde{S} \rightarrow S$, such that for every $h \in S$ there exists an open neighborhood $U$ of $p$ such that $h^{-1}(U)$ (the inverse image of $U$ under $h$ ) is a disjoint union of open sets in $\tilde{S}$, each of which is mapped homeomorphically onto $U$ by $h$. The map $h$ is called the covering map. A connected covering space is a universal cover if it is simply connected. Suppose $\gamma \subset S$ is a loop through the base point $p$ on $S$. Let $\tilde{p}_{0} \in \tilde{S}$ be a pre-image of the base point $\tilde{p}_{0} \in h^{-1}(p)$, then there exists a unique path $\tilde{\gamma} \subset \tilde{S}$ lying over $\gamma($ i.e. $h(\tilde{\gamma})=\gamma$ ) and $\tilde{\gamma}(0)=\tilde{p}_{0} . \tilde{\gamma}$ is a lift of $\gamma$.

A deck transformation of a cover $h: \tilde{S} \rightarrow S$ is a homeomorphism $f: \tilde{S} \rightarrow \tilde{S}$ such that $h \circ f=h$. All deck transformations form a group, the so-called deck transformation group. A fundamental domain of $S$ is a simply connected domain, 
which intersects each orbit of the deck transformation group only once. A fundamental domain can be obtained by slicing a surface $S$ open along a canonical fundamental group generators as shown in figure 3(b), where a finite portion of the universal cover of a genus two surface is shown, different fundamental domains are encoded by different colors in (b) and (c). Deck transformations map fundamental domains to fundamental domains. The deck transformation $\operatorname{group} \operatorname{Deck}(S)$ is isomorphic to the fundamental group $\pi_{1}(S, p)$. Let $\tilde{p}_{0} \in h^{-1}(p), \phi \in \operatorname{Deck}(S), \tilde{\gamma}$ is a path in the universal cover connecting $\tilde{p}_{0}$ and $\phi\left(\tilde{p}_{0}\right)$, then the projection of $\tilde{\gamma}$ is a loop on $S, \phi$ corresponds to the homotopy class of the loop $\phi \rightarrow[h(\tilde{\gamma})]$. This gives the isomorphism between $\operatorname{Deck}(S)$ and $\pi_{1}(S, p)$.

6.3. Poincaré Disk Model. In this work, we use Poincaré disk to model the hyperbolic space $\mathbb{H}^{2}$, which is the unit disk $|z|<1$ on the complex plane with the metric

$$
d s^{2}=\frac{4 d z d \bar{z}}{(1-z \bar{z})^{2}}
$$

The rigid motion is the Möbius transformation

$$
z \rightarrow e^{i \theta} \frac{z-z_{0}}{1-\bar{z}_{0} z}
$$

where $\theta$ and $z_{0}$ are parameters. A hyperbolic circle with center $\mathbf{c}$ and radius $r(\mathbf{c}, r)$ is also a Euclidean circle $(\mathbf{C}, R)$ with

$$
\mathbf{C}=\frac{2-2 \mu^{2}}{1-\mu^{2} \mathbf{c} \overline{\mathbf{c}}} \mathbf{c}, R^{2}=\mathbf{C} \overline{\mathbf{C}}-\frac{\mathbf{c} \overline{\mathbf{c}}-\mu^{2}}{1-\mu^{2} \mathbf{c} \overline{\mathbf{c}}}
$$

where $\mu=\tanh \frac{r}{2}$.

Given two points $p$ and $q$ on $\mathbb{H}^{2}$, the unique geodesic (hyperbolic line) through them is a circular arc and is perpendicular to the unit circle.

Acknowledgments. This work is partially supported by LouisianaBoard of Regents Fund: (NSF)LEQSF-RD-A-21.

\section{REFERENCES}

[1] P. Shilane, P. Min, M. Kazhdan, and T. Funkhouser, The princeton shape benchmark, in: SMI: Proceedings of the Shape Modeling International, 2004, pp. 167-178.

[2] N. Iyer, S. Jayanti, K. Lou, Y. Kalyanaraman, and K. Ramani, Three-dimensional shape searching: state-of-the-art review and future trends, Computer-Aided Design, 37:5(2005), pp. 509-530.

[3] J. W. Tangelder and R. C. Veltkamp, A survey of content based $3 d$ shape retrieval methods, in: Multimedia Tools and Applications, in press, 2008.

[4] X. Gu And S.-T. Yau, Surface classification using conformal structures, in: ICCV '03: Proceedings of the Ninth IEEE International Conference on Computer Vision, 2003, p. 701. 
[5] M. Jin, F. LuO, S.-T. YAU, AND X. GU, Computing geodesic spectra of surfaces, in: SPM '07: Proceedings of the 2007 ACM symposium on Solid and physical modeling, 2007, pp. 387-393.

[6] S. Wang, Y. Wang, M. Jin, X. Gu, And D. Samaras, 3d surface matching and recognition using conformal geometry, in: CVPR '06: Proceedings of the 2006 IEEE Computer Society Conference on Computer Vision and Pattern Recognition, 2006, pp. 2453-2460.

[7] X. Gu, S. Wang, J. Kim, Y. Zeng, Y. Wang, H. Qin, and D. Samaras, Ricci flow for $3 d$ shape analysis, in: ICCV '07: IEEE International Conference on Computer Vision (ICCV'07), 2007, pp. 1-8.

[8] F. LuO, Geodesic length functions and teichmüller spaces, J.DIFFERENTIAL GEOMETRY, 48(1998), p. 275.

[9] M. Jin, W. Zeng, F. Luo, And X. Gu, Computing teichmüller shape space, IEEE Transactions on Visualization and Computer Graphics, 15:3(2009), pp. 504-517.

[10] M. Ben-Chen and C. Gotsman, Characterizing shape using conformal factors, in: Proceedings of Eurographics Workshop on Shape Retrieval, 2008.

[11] M. Reuter, F. Wolter, and N. Petnecke, Laplacespectra as fingerprints for shape matching, in: Solid and Physical Modeling, 2005, pp. 101-106.

[12] R. Rustamov, Laplace-beltrami eigenfunctions for deformation invariant shape representation, in: Proc. Symposium on Geometry Processing, 2007.

[13] P. Xiang, C. Hua, F. Gang, and Z. Chuan, Pose insensitive $3 d$ retrieval by poisson shape histogram, Lecture Notes in Computer Science, vol. 4488, pp. 25-32, 2007.

[14] X. Gu And B. VemurI, Matching 3d shapes using 2d conformal representations, in: MICCAI (1), 2004, pp. 771-780.

[15] Y. Wang, M.-C. Chiang, and P. M. Thompson, Mutual information-based 3d surface matching with applications to face recognition and brain mapping, in: ICCV '05: Proceedings of the Tenth IEEE International Conference on Computer Vision (ICCV'05) Volume 1, 2005, pp. 527-534.

[16] V. Jain AND H. Zhang.

[17] A. Elad AND R. Kimmel, On bending invariant signatures for surfaces, IEEE Transactions on Pattern Analysis and Machine Intelligence, 25:10(2003), pp. 1285-1295.

[18] T. Tung And F. Schmitt, The augmented multiresolution reeb graph approach for contentbased retrieval of $3 d$ shapes, International Journal of Shape Modeling, 11:1(2005), pp. 91-120.

[19] R. Gal, A. Shamir, And D. Cohen-or, Pose oblivious shape signature, IEEE Transactions on Visualization and Computer Graphics, 13:2(2007), pp. 261-271.

[20] J. Munkres, Elements of Algebraic Topology.

[21] W. Thurston, Three-Dimensional Geometry and Topology.

[22] P. Buser, Geometry and spectra of compact Riemann surfaces.

[23] A. Hatcher, P. Lochak, And L. Schneps, On the teichmüller tower of mapping class groups, J. Reine Angew. Math., 521(2000), pp. 1-24.

[24] E. C. D. Verdière And F. Lazarus, Optimal pants decompositions and shortest homotopic cycles on an orientable surface, J. ACM, 54:4(2007), p. 18.

[25] T. K. Dey, K. Li, J. Sun, And D. Cohen-Steiner, Computing geometry-aware handle and tunnel loops in $3 d$ models, ACM Trans. Graph., 27:3(2008), pp. 1-9.

[26] X. LI, X. Gu, AND H. QIN, Surface mapping using consistent pants decomposition, IEEE Transactions on Visualization and Computer Graphics, 2008.

[27] В. Chow And F. Luo, Combinatorial Ricci flows on surfaces, Journal Differential Geometry, 63:1(2008), pp. 97-129.

[28] M. Jin, J. Kim, F. Luo, And X. Gu, Discrete surface ricci flow, IEEE Transaction on Visualization and Computer Graphics, 14:5(2008). 
MIAO JIN ET AL. 